\title{
PROTON- AND $\alpha$-RADIATION OF THE MICRO-PINCH WITH THE BORON-CONTAINING TARGET
}

\author{
Anatolit A. Gurin ${ }^{a, b, *}$, Andrit S. Adamenko ${ }^{a}, \operatorname{Stanislav~V.~Adamenko~}^{a}$, \\ MYKOLA M. KUZMENKO ${ }^{a}$
}

${ }^{a}$ EDL Proton-21 Ltd, Kyiv, Ukraine

${ }^{b}$ Institute for Nuclear Research NASU, Kyiv, Ukraine

* corresponding author: gurin@kinr.kiev.ua

\begin{abstract}
Using ion pinhole camera and track detectors, the image of hot spot is recorded in a pulsed diode micro-pinch equipped with a solid anode target. The track image is a record of repeated fronts of fast protons with energies up to $1 \mathrm{MeV}$. Fluctuations in the ion luminosity of hot spot are associated with the wave-like nature of the proton accelerating processes in the dense plasma of target material, which is characterized by a mean energy of $100 \mathrm{keV}$. The results of the track analysis of a fast ions, detected in the Thomson analyser in experiments with boron-polyethylene targets, are presented. In $5 \%$ of the shots, the presence of $\alpha$-particles of energy up to $2 \mathrm{MeV}$ in the flux of fast ions is discovered by means of Thomson analyser equipped with track detectors. Estimations of total amount of helium nuclei as products of nuclear reactions $\mathrm{p}\left(\mathrm{B}_{11}, 2 \alpha\right) \alpha$ result in an output of $10^{8} \div 10^{9}$ per successive shot.
\end{abstract}

KEYWORDS: micro-pinch, fast ions, proton, boron, target, $\alpha$-particles.

\section{INTRODUCTION}

Hot spot, flashing in the channel of micro-pinch ("plasma spark"), is an intriguing object since when as early as the fifties of last century it was shown that the "star", helium and hydrogen-like emission spectra of heavy elements up to iron can be simulated in laboratory experiments. In micro-pinches arising from the high-voltage breakdown of the vacuum diode, the temperature exceeds $10 \mathrm{keV}$, and the plasma density is close to the solid state. However, the uncontrolled nature of self-organization is the main obstacle to the use of micro-pinches for controlled fusion. Breakdowns in vacuum diodes prepared by anodic pre-plasma formed as a result of the surface ionization of the anode by the primary electron beam, or by different means, with the parameters of the beam and anode plasma are critical for the formation of the hot spot. This picture is much more complex one that takes place in the gas-filled low-pressure "plasma focus", which has long maintained a leading position among the number of fusion projects in the $\mathrm{D}-\mathrm{D}$ neutron output (notice the surveys in this topic $[9,5$ ).

In the EDL "Proton-21", we found new and interesting features of the hot spots. In a vacuum diode sparked as the load of the plasma opening switch at a voltage of $400 \mathrm{kV}$, with the current rise of tens nanoseconds up to values of $30 \div 40 \mathrm{kA}$, there is a phenomenon which can be described as flicker luminosity of the hot spot, but only in terms of ion optics. Ion pinhole-camera fitted with track detector recorded a repetition of the fronts of fast protons, which characterize the high-energy corpuscular radiation of micropinch. Figure 1 shows an example of the hot spot image, which, in image of "plasma focus" or "laser focus" 8, 10, as a compact spot, is a composite image in the form of several strips ("candles") oriented along the pinch axis. Separate sparse track clusters are sometimes recorded too. (The pinhole axis is placed in plane of the diode, the distance from the diode axis to the inlet is $17 \mathrm{~mm}$ and the distance to the detector $45 \mathrm{~mm}$, i.e. zoom factor is about 2). Width of track strips $(200 \div 220 \mu \mathrm{m})$ is weakly dependent on the diameter of the inlets (tens $\mu \mathrm{m}$ ), so the geometrical considerations of to determine hot spot size about $100 \mu \mathrm{m}$.

"Heads" of strips, its close to the pinhole axis edges, are formed by deep latent tracks of fast protons with energies from 0.6 to $1 \mathrm{MeV}$, while the continuation of the strips represented highly degraded tracks down to the bottom of track formation energy $100 \mathrm{keV}$. The images, and all of their details recorded by the pinhole, are not snapshots of hot spot but the integral pictures without any time binding. However, the main conclusions can be made about how such images arise. Overlapping of the strips shows that the flows of fast protons are emitted not simultaneously but in a number of fronts following sequentially. The full picture occurs as a result of drawing compact spot, which is the trace of a thin radial beam of protons penetrating into the pin-hole camera during a period of each front. Each strip is formed by deflection of slow protons of the passing front in the peripheral azimuth magnetic field to be present in the pin-hole camera.

Images like in Fig. 1 has been published previously [7, 1]. Since hundreds of images similar to Fig. 1 have been accumulated, as well as the energy spectrum [7] is confirmed. In our experiments, the average energy of track-productive protons is within 


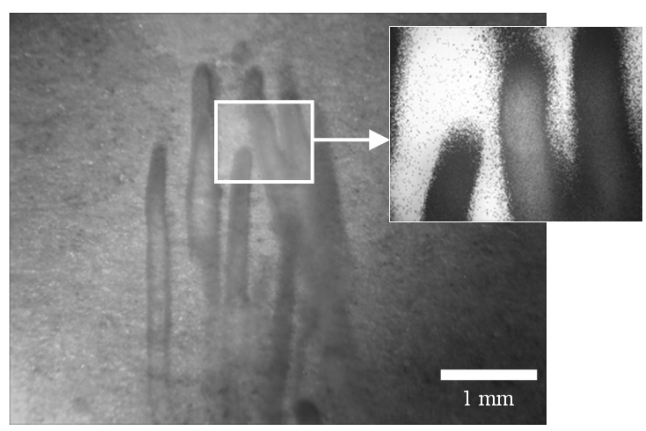

Figure 1. The typical image of the "hot spot" of micro-pinch on the track detector exposed in the ion pinhole camera.

the range $200 \div 300 \mathrm{keV}$. Total number of fast protons in each of the shots is valuated at the amount of $10^{14} \div 10^{15}$ per shot. Note, the usual optical radiation of the diode discharge, which continues microseconds after fast proton passing, is realized with the amount order of $10^{18}$ hydrogen atoms coming from the peripheral anode plasma.

In our experiments, the "target" is an element of the anode - a rod about $1 \mathrm{~mm}$ thick, fastened in the metal anode in the diode gap. Characteristically, fully equally well be used as a metal or dielectric targets, which clearly indicates that the target material provides rise, above all, a conductive anode plasma. Despite the long history of the problem, there is still no sufficiently complete model of the mechanisms of the hot spot and the proton acceleration in micropinches. The concept of collective ion acceleration with virtual cathode can be considered as a dominant [5]. However, we note the 2] where, in the configuration of the "Luce diode" close to which we used, many bursts of accelerated protons were found, the possibility of its acceleration with the potential hole of electrons was refuted, as well the wave character of the formation of accelerated proton streams was suggested. We also note [3], which also mentions the radiation of separate bunches of protons from a micro-pinch. In our view, the most important for understanding the processes of proton acceleration results are obtained in [13, 12, where the effect of predominant acceleration of protons as the easiest component of the plasma is observed, followed by the shock wave of the magnetic field, which forms a strong current channel. This mechanism brings to the multispecies Hall MHD model, waiting for further development, of acceleration and separation of ions due to fast magnetic field penetration in plasma with current rising. Results [13, 12] relate to the current-carrying plasma in erosion switches, but it is natural to assume that the same sharpened processes take place in the more dense micro-pinch plasma after the breakdown of the diode as a shunt of plasma switch.

In this publication we would like to note that Fig. 1 and other data confirm the wave-like nature of the proton accelerating processes directly in a dense plasma of hotspot. The track images on detectors indicate that in some cases there are up to 20 "candles", though the most high energy emission founded in the case of a small number of them, from 3 to 5, as shown in Fig. 1. If the accelerated proton fluxes arise directly inside the hot spot of micro-pinch, presence of relative motion of protons through the relatively dense plasma of heavy ions is an important attribute of the hot spot. Although there is still no complete dynamical model, it is reasonably to conclude that the relative velocity of protons, which are involved in the process of pinching and radiation, is scaled by the average energy order of $100 \mathrm{keV}$ recorded by tracks in the pinhole, at the finish of proton free movement. The ensemble of these protons could be characterized by the time-averaged velocity distribution function with temperature scale of $100 \mathrm{keV}$. These temperatures do not occur in nature, is more correct to speak of, rather, a hybrid version of the beam-plasma system, attractive in terms of ion inertial fusion.

The purpose our experiments was to examine the possibility of occurrence of a number of fusion reactions $\mathrm{p}+\mathrm{B}_{11}=3 \alpha+8.7 \mathrm{MeV}$ in the "hot spot", initiated in the micro-pinch discharges with solid boroncontaining anode targets. This reaction has long been discussed as the most attractive from the standpoint of the requirements of environmentally friendly, "aneutronic" energy of the future (" $3 \alpha$-energetics", as some authors). It is extremely interesting to determine whether achieved in the ongoing laboratory experiments the conditions for this reaction, which is also known that the energy threshold is very high (about $500 \mathrm{keV}$ ) and is irresistible to all contemporary fusion projects.

Getting to experiment with boron-containing targets, we are fully aware that the parameters of our micro-pinch are not sufficiently high for to achieve the proton-boron above-barrier synthesis, so one can not expect to see plenty of the presence of alpha particles in the products affected by the imploded targets. The exponentially decreasing spectrum of fast protons, while the average energy in the range $200 \div 300 \mathrm{keV}$, only allows for the possibility of individual and subbarrier reactions. However, results of first steps in this direction may be of interest.

\section{EQUIPMENT AND METHODS}

The preparation of targets based on boron is impeded with the fact that pure natural boron as well the main boron-hydrogen compounds do not form a solid state. Therefore, the target used on the basis of hydrogenrich filler, as is used most often pure polyethylene, containing a suspension of amorphous natural boron $\left(83 \%\right.$ of $\left.\mathrm{B}_{11}\right)$. It was possible to achieve weight ratio of 8:5 in favour of boron. We also used other fillers: paraffin, silica or epoxy glue. Used also coating targets: a gold foil (submicron natural "gold leaf"), the enrichment of the surface layer with the amorphous boron. About 200 experiments have been performed. 


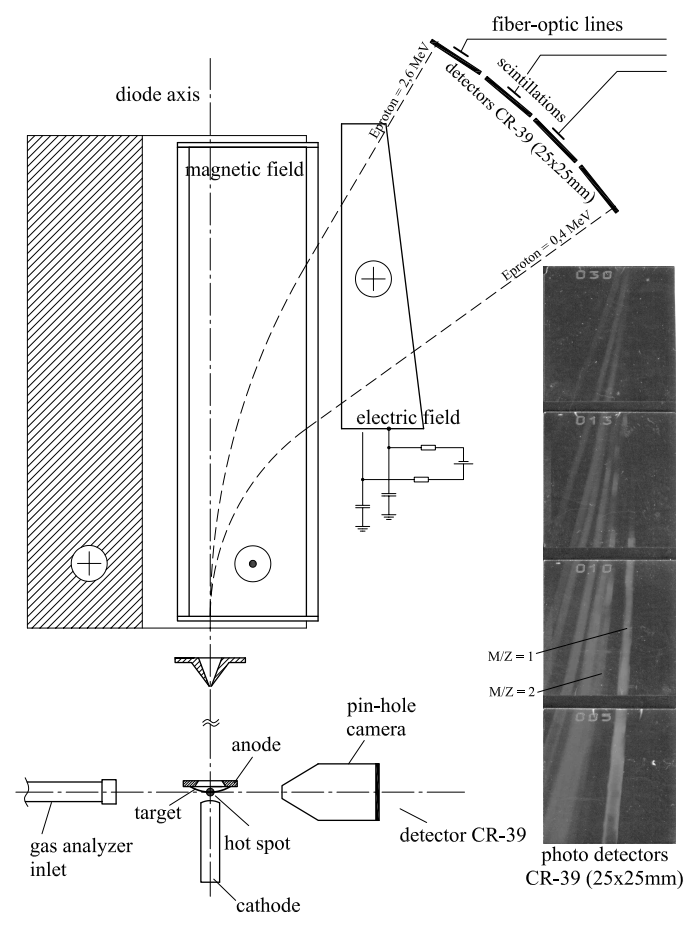

Figure 2. Experimental arrangement; also the example of photo image of Thomson parabolas on the etched track detectors is shown.

To register the track pin-hole image in the pin-hole camera the target was oriented parallel to the axis of the diode. We also used diagnostics, which is shown in Fig. 2, where the target was located across the anode axis before the $6 \mathrm{~mm}$ hole in the flat anode letting out a beam of ions into the space behind the anode. Then a thinned plasma beam passes sequentially the parallel magnetic $(9 \mathrm{kGs})$ and electric $(6 \mathrm{kV}$ on planar electrodes with a gap of $8 \mathrm{~mm}$ ) fields according to scheme of an Thomson analyser. Figure 2 observes proportions of the target, fields and the proton orbits sizes. In our Thomson analyser, ions are registered with track detectors and scintillators provided with electronic optic signal conversion. Scintillators settled in several positions that meet certain energy of protons, and allows to measure time of flight signals of fast ions on the base $69 \mathrm{~cm}$. In Fig. 3 the waveform of protons signal delay is registered by scintillator in position $E_{\text {proton }}=0.5 \mathrm{MeV}$, together with the diode current waveform recorded by Rogowski coil, as well the X-ray electron beam bremsstrahlung signal detected by the scintillator open only for hot spot. The delay time of protons corresponds with their emission at growth phase of the discharge current lasting no more than $40 \mathrm{~ns}$. (These data will be published in more details elsewhere).

Time of flight measurements are insufficient for the final identification of the ion composition of fast plasma flows of the affected target. To this end, the tracks of ions recorded on the track detectors CR-39 along the Thomson parabolas $M / Z=1$

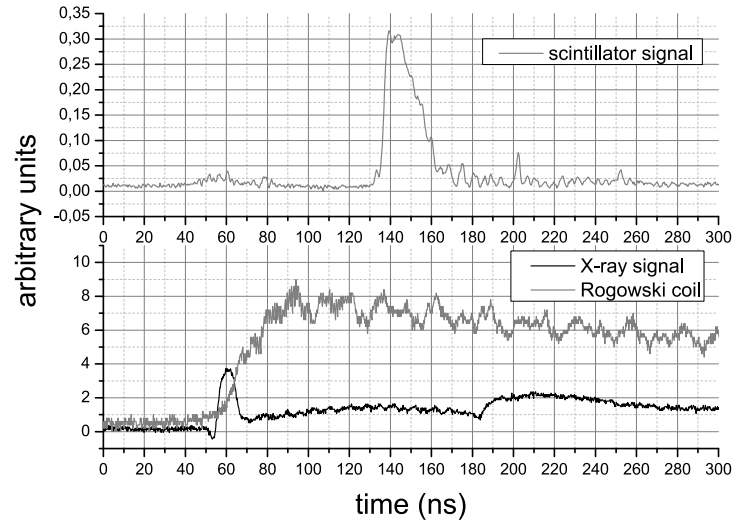

Figure 3. The waveforms of diode current, collimated X-ray hot-spot emission and signal of scintillator placed at position $E_{\text {proton }}=0.5 \mathrm{MeV}$ on proton parabola $M / Z=1$ in Thomson analyser.

(of the protons) and $M / Z \geq 2$ (of more heavy ions) were studied. In our experiments the ions $\mathrm{He}_{4}^{+2}, \mathrm{~B}_{10}^{+5}$, $\mathrm{B}_{11}^{+6}, \mathrm{C}_{12}^{+6}$, etc, can occupy the $M / Z=2$ parabola (the isotope splitting of parabolas is not achieved). In successful shots, in which second parabola is well expressed, the track groups were selected within its area for to be analysed by the "squared diameters asymptotic method" (SDAM) [1, 11], which allows to determine the full track length in the detector, $R$, and the maximal depth of etched tracks, $L$, by measuring the diameters of the tracks in the normal procedure a long time etching. These data allow us to determine the average reduced rate, $V=R /(R-L)$, and to compare these values with the calibration and the theoretical dependences $V(R)$ for different ions. This procedure reduces to the construction of the locus for all analysed tracks, " $V$ vs $R$ ", and to fixation of splitting loci in accordance with the calibration or theoretical data. We used the theoretical and experimental dependences $V(R)$ 4, 6], and the gauge locus for alphaparticles [7, which we built for $\alpha$-tracks on the detector irradiated with neptunium source $\left(\mathrm{Np}_{247}\right)$.

The main drawback is the large SDAM time consuming, the need for high-precision optical measuring instruments, especially in the case of short range tracks of heavy ions. However, SDAM is convenient, or even the only possible method for finding and determining the energy of alpha particles $i n$-situ, in a mixed flow of ions very low energy light nuclei with a range $5 \div 10 \mathrm{~mm}$ in the detector, for which the values of $V$ are well distinguished.

The following data also illustrates another feature of SDAM. As a rule, especially in the case of heavy ions, the calculated error of $V, R$ for the individual tracks are small compared with the statistical spread of these values for dense groups of similar tracks selected for analysis. Random parameters of individual tracks (Brownian struggling), which the SDAM ignores, affect the final form of loci. Therefore, in Figs. 46 data of some groups of tracks with close values of $R$ combined into a single "middle track" 


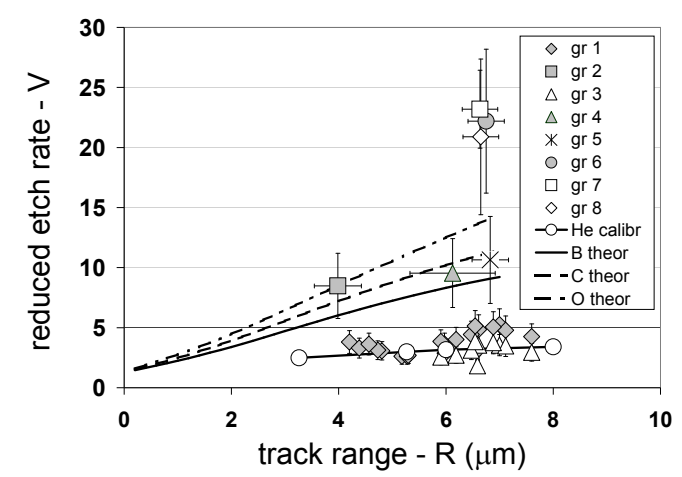

Figure 4. Locus 80 tracks chosen for the analysis within different parts of the parabola $M / Z=2$; groups number 1 and 3 represent 30 individual tracks of $\alpha$ particles with showing $20 \%$ evaluative errors of $V$ measurements; groups number 2 and 4-8, join each 10 into an 'average' track and belong apparently to the ions $\mathrm{B}, \mathrm{C}$ or $\mathrm{O}$, and even heavier ions.

followed by the standard deviation of coordinates $(\Delta R$, $\Delta V)$ for the group. This helps the interpretation of measurement results. We note in this connection that in 4] data, were used to calibrate the relations $(R, L)$, are presented as a average statistical values of 12 tracks, with no indication of error intervals. Below the loci are showing standard deviation $\Delta V, \Delta R$ for the respective groups.

\section{RESUlts}

Summarized results of these studies are as follows. In some shots within the parabola $M / Z=2$ separate track groups are recorded, which are identified as $\alpha$-particles of range $8 \div 4 \mu \mathrm{m}$ in the detector, which corresponds to a range of energy $\alpha$-particles of $0.8 \div 1.7 \mathrm{MeV}$, and single tracks with a range up to 14 microns. Only about 10 experiments in a series of 200 shoots were positive. In some cases, track analysis for various reasons do not allow definite conclusions. Figures 46 are positive examples of identification of $\alpha$-particles as well as an achieved accuracy of the construction of loci by the SDAM. This data is presented together with the gauge $V(R)$ for $\alpha$-particles. We also give the theoretical relations $V(R)$ for light nuclei, as in the case of small length of heavy nuclei any calibration are absent.

Figures 4, 5 show the splitting of loci indicating the presence of $\alpha$-particles with energies up to $1.7 \mathrm{MeV}$ in the flow of heavier ions. Note that in the ranges $R<4 \mu \mathrm{m}$ track analysis is difficult, and the SDAM does not allow unambiguous identification of such very weak tracks. Figure 6 combines the results of detecting relatively small groups of $\alpha$-tracks, including the case of registration of the fastest individual $\alpha$-particles: $3 \alpha$-tracks are characterized by runs about 13 microns, which corresponds to the energy $\alpha$-particles of $2.7 \mathrm{MeV}$. Note that the single $\alpha$-tracks with big runs are often recorded in arbitrary samples, however such data can hardly be considered conclusive.

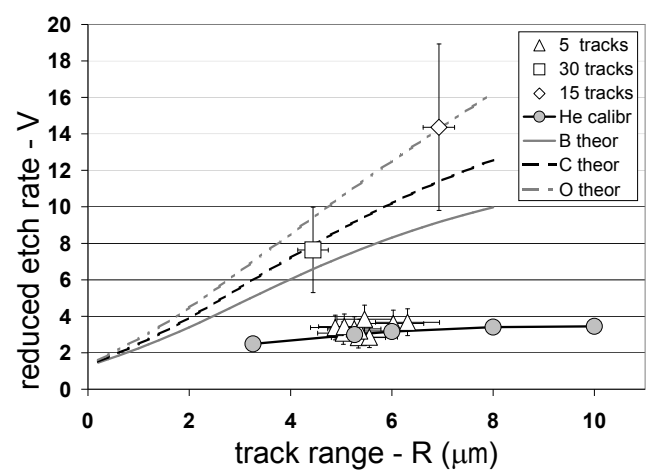

Figure 5. Locus of almost 100 tracks (boron-plastic target coated with $\mathrm{Au}$ ): a rich locus $10^{5}$ tracks belong to $\alpha$-particles, two group loci are treated equally as belonging to the nuclei of carbon or oxygen.

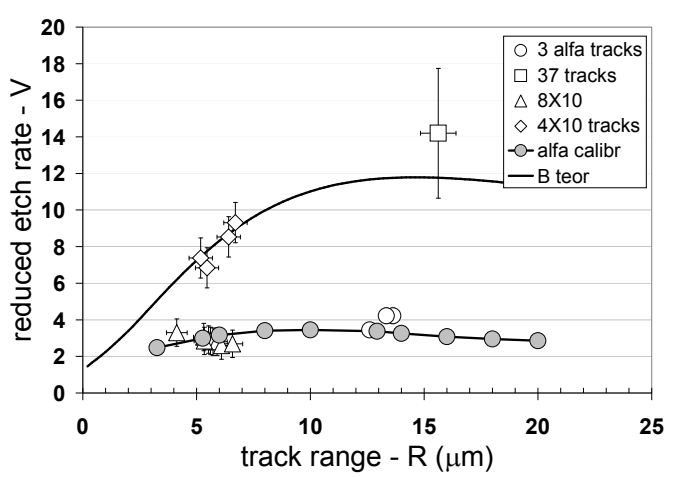

Figure 6 . The total loci of 120 tracks of the 4 experiments, each of which $\alpha$-particles tracks are detected, including recorded the fastest $\alpha$-particles in one experiment: three tracks on the background of 37 tracks of boron nuclei.

\section{Discussion And CONCLUSiOnS}

These results indicate that we have achieved accuracy is sufficient for the identification of alpha particles, as the lightest nuclei corresponding to the condition $M / Z=2$. The main reason for further discussion is the absence of alpha particles in the range $3 \div 4 \mathrm{MeV}$, as products of the reaction $\mathrm{p}\left(\mathrm{B}_{11}, 2 \alpha\right) \alpha$. The identification of such particles would be more easy task. Another question is their amount.

Obviously, the detectors recorded the helium ions formed in the central axial region of the anode target, which, when explosions boron-hydrocarbon target is the source of the dense plasma, the starting density is close to the solid. In this case the energy of $8.7 \mathrm{MeV}$, the resulting "harrow-proton" reaction, being divided onto three $\alpha$-particles, is partially lost in the target material. Note that the range of $3 \mathrm{MeV} \alpha$-particles in hydrocarbon media does not exceed 20 microns. The detectors record only a portion of the emitted fast ions of helium, have overcome the scattering in the target material, whereas some of the ions of helium is completely inhibited, and mixed with the gas-plasma decay products of the target (data of gas analyser confirm such conclusion). Such an effect may occur if the "op- 
tically opaque" core of the hot spot (in terms of ion optics) has a size of $100 \mu \mathrm{m}$.

The determination of the total number of fast helium ions in the plasma flows of affected targets directly in the experiment, in contrast to the analogous problem for the neutron experiments with DD inertial fusion, seems to be much more difficult. The total number of registered alpha-particles is difficult to assess because the complete overlapping of etched tracks makes them impossible to be counted. $\alpha$-particles are found only in that part of the parabola $M / Z=2$, which corresponds to the interval $1 \div 2 \mathrm{MeV}$, and the number of tracks is about $1 \%$ of the total track number on the corresponding segment of the parabola. A comparison of the intensities of the first and second parabolas shows that the coefficient is not higher $10^{-6}$ can be taken to evaluate the proportion of the total number of fast helium ions to the total number of fast protons are regularly recorded. An integral number of fast protons emitted by the target is estimated about $10^{14} \div 10^{15}$. (This number is determined by counting the total number of proton tracks, deflected by the magnetic field, directly in the magnet gap with an additional small $5 \mathrm{~mm}$ aperture at the entrance into magnet). Consequently, up to $10^{8}$ fast helium ions can be produced in a lucky shot with boronpolyethylene target. It must be recognized that the reproducibility of the process is low: only $5 \%$ of the experiments gave a positive result.

This estimation of the amount of helium produced in the experiments with boron-containing targets is confirmed, and even rises up to a value $10^{9}$ per shot, thanks to more reliable data of the gas analyser, based on samples taken from the chamber immediately after the shooting. These samples also contain helium atoms, which are formed as a result of complete inhibition of alpha-particles in the diode plasma and are not registered by the Thomson analyser.

The above results of the track detection help to do, rather, a qualitative conclusion about the presence of the nuclear reaction $\mathrm{p}\left(\mathrm{B}_{11}, 2 \alpha\right) \alpha$ in the hot spot of laboratory micro-pinch with not a record-breaking parameters. Note that in the early experiments on thermonuclear D-D fusion using "plasma focus" number of neutrons recorded at $10^{8}$ per shot was considered as a relative success. In experiments with solid targets micro-pinch on program " 3 - $\alpha$-energy" must take the next step: to achieve the value of the average proton energy order of $1 \mathrm{MeV}$ in fluctuations of corpuscular emission of hot spots. If the average energy of a pulsed "runaway" of protons will be so high, it can be expected that the "flicker" will be associated with abundant above-barrier proton-boron synthesis in the hot spot plasma.

\section{REFERENCES}

[1] S. V. Adamenko, A. S. Adamenko, A. A. Gurin, Yu. M. Onishchuk. Track measurements of fast particle streams in pulsed discharge explosive plasma. Radiation measurements 40(2-6):486-489, 2005.

[2] R. Adler, J. A. Nation, V. Cerlin. Collective acceleration of protons in relativistic electron beam propagation in evacuated drift tubes. Phys Fluids 24(2):347-356, 1981.

[3] V. M. Bystritski, A. N. Didenko, Ya. E. Krasik, et. al. . Letters to JETP 4(9):547-551, 1978

[4] B. Dorschel, D. Hermsdorf, K. Kander, H. Kuchne. Track parameters and etch rates in alpha-irradiated CR-39 detectors used for dosemeter response calculation. Radiat Prot Dosim 78(3):205-212, 1998.

[5] A. E. Dubinov, I. Yu. Kornilova, V. L. Selemir. Collective ion acceleration in systems with a virtual cathode. Advances in Physical Sciences 45(11):1109-1130, 2002.

[6] A. N. Golovchenko. On registration properties of intercast company CR-39. Int J of Radiat Appl $\mathcal{E}$ Instrum Part D 20(3):517-519, 1992.

[7] A. A. Gurin, A. S. Adamenko. Fundamental theories in physics. Controlled nucleosynthesis. Springer, 2007. Pages 105-151. Edited by S. Adamenko, F. Selleri, A. van der Merve.

[8] U. Jager, H. Herold. Fast ion kinetics and fusion reaction mechanism in the plasma focus. Nucl Fus 27(3):407-423, 1987.

[9] R. B. Miller. An introduction to the physics of intense charged particle beam. Plenum Press, N.-Y., L., 1984.

[10] D. C. Slater. Pinhole imaging of fast ions from laser-produced plasmas. Appl Phys Lett 31(3):196-198, 1977.

[11] G. Somogyi, S. A. Szalay. Track-diameter kinetics in dielectric track detectors. Nucl Instrum and Methods 109(2):211-232, 1973.

[12] S. B. Swanekamp, R. J. Commisso, P. F. Ottinger, et al. Species separation and field-penetration in a multi-component plasma. In BEAMS 2002: 14th International Conference on High-Power Particle Beams, pp. 455-458. Albuquerque, New Nexico (USA), 2002.

[13] A. Weingarten, R. Arad, Y. Maron, A. Fruchman. Ion separation due to magnetic field penetration into a multispecies plasma. Phys Rev Lett 87(11):115004, 2001. 